\title{
IQC analysis of reset control systems with time-varying delay
}

DOI:

10.1080/00207179.2018.1426881

\section{Document Version}

Accepted author manuscript

Link to publication record in Manchester Research Explorer

\section{Citation for published version (APA):}

Mercader, P., Carrasco, J., \& Baños, A. (2018). IQC analysis of reset control systems with time-varying delay. International Journal of Control. https://doi.org/10.1080/00207179.2018.1426881

\section{Published in:}

International Journal of Control

\section{Citing this paper}

Please note that where the full-text provided on Manchester Research Explorer is the Author Accepted Manuscript or Proof version this may differ from the final Published version. If citing, it is advised that you check and use the publisher's definitive version.

\section{General rights}

Copyright and moral rights for the publications made accessible in the Research Explorer are retained by the authors and/or other copyright owners and it is a condition of accessing publications that users recognise and abide by the legal requirements associated with these rights.

\section{Takedown policy}

If you believe that this document breaches copyright please refer to the University of Manchester's Takedown Procedures [http://man.ac.uk/04Y6Bo] or contact uml.scholarlycommunications@manchester.ac.uk providing relevant details, so we can investigate your claim.

\section{OPEN ACCESS}




\title{
IQC analysis of reset control systems with time-varying delay
}

\author{
P. Mercader ${ }^{\mathrm{a}}$, J. Carrasco ${ }^{\mathrm{b}}$ and A. Baños ${ }^{\mathrm{a}}$ \\ a Dpt. Informática y Sistemas, University of Murcia, Spain; \\ b Control Systems Centre, School of Electrical and Electronic Engineering, The University of \\ Manchester, UK
}

\begin{abstract}
ARTICLE HISTORY
Compiled November 15, 2017

ABSTRACT

The study of input-output stability of reset control systems with time-varying delay is addressed in this work. The time-varying function that defines the delay is assumed to be bounded on magnitude and variation. This approach also covers the particular case of constant time delay, but it is studied separately to obtain less conservative results. After proposing a convenient loop transformation, the stability analysis is performed by means of the integral quadratic constraint (IQC) framework. Then by applying the Kalman-Yakubovich-Popov (KYP) Lemma, easily checkable conditions in form of linear matrix inequalities (LMI) are obtained. To conclude, some numerical examples are provided illustrating the proposed criteria.
\end{abstract}

\section{KEYWORDS}

Time-delay systems; Reset control systems; Integral quadratic constraint.

\section{Introduction}

A reset controller is a linear system whose states are set to zero (reset actions) when its input equals zero, or more generally, when it satisfies a given condition. This control technique was one of the first attempts to overcome fundamental limitations of linear and time-invariant (LTI) control systems (Clegg, 1958; Horowitz \& Rosenbaum, 1975; Krishman \& Horowitz, 1974). An example of a reset control system reaching specifications that are not achievable for any LTI controller is presented by Beker, Hollot, and Chait (2001). Some intuition about its capabilities may be gained from the describing function approach. It shows that reset controllers are able to yield a similar gain characteristic of a given LTI controller, but with significantly less phase lag. The latter property makes reset control an attractive strategy to deal with time-delayed systems.

A well-known caveat of this control strategy is that the mere addition of reset actions may destabilise a stable control system. This issue has motivated many works. The problem of the internal stability of reset control systems with constant time delay has been thoroughly studied, see, for example, (Baños \& Barreiro, 2009; Barreiro \& Baños, 2010; Davó \& Baños, 2013; Davó, Gouaisbaut, Baños, Tarbouriech, \& Seuret, 2015; Mercader, Davó, \& Baños, 2013). Nevertheless, the aspect of input-output stability and issues like time-varying delay and alternative reset laws remain virtually unexplored. An inherent difficulty when studying the stability of time-delayed reset 
control systems is the presence of discontinuities in the state (or possibly some states) of the reset controller (see Davó and Baños (2013)). That makes difficult to rigorously study the stability by adopting a Lyapunov-Krasovskii approach, thus calling for an input-output approach. Moreover, the problem becomes even harder to solve in the case of time-varying delay and/or alternative reset laws. These facts motivate the use of an alternative approach able to circumvent these difficulties. The approach adopted here is the IQC framework exploiting input-output properties of reset controllers.

Concerning to IQC analysis, it should be noted that in the last two decades robust control theory has been reformulated within the IQC framework (Megretski \& Rantzer, 1997), which gives an unifying framework for problems in modern robust control. IQCs are very useful in capturing a rich class of uncertainties. For the particular case of time-delay, it has been considered as a type of structured uncertainty in a number of works, see, e.g. (Kao \& Rantzer, 2007; Pfifer \& Seiler, 2015; Tugal, Carrasco, Falcon, \& Barreiro, 2016).

This work studies input-output stability problem of reset control systems with timevarying delay using the IQC approach. We combine the reset controller and the delay as a MIMO nonlinearity, and use the input-output properties of reset systems (Carrasco, Baños, \& van der Schaft, 2010; Forni, Nešić, \& Zaccarian, 2011). Applying then the IQC theorem, stability conditions for this class of systems are obtained. Preliminaries results were presented by Mercader, Carrasco, and Baños (2013). To the best of our knowledge, this approach provides the first input-output stability conditions for reset control systems with time-varying delay via IQC framework. We note that discontinuity in the output signal is not a limitation in the IQC framework. Indeed, passivity properties have been already shown by Carrasco et al. (2010) and Forni et al. (2011). The IQC framework is used to combine these passivity properties with the time delay properties developed in Kao and Rantzer (2007). Other authors, e.g. Fetzer and Scherer (2016), use IQC framework for the sample and hold operator.

This work starts giving some preliminary background in Section 2. Section 3 presents the time-delay reset control system. Stability criteria for time-delay reset control systems are presented in Section 4. Applications of the proposed criteria are given in Section 5. Finally, some conclusions are drawn.

Notation: $\mathbf{x} \in \mathbb{R}^{n}$ is a column vector, $|\mathbf{x}|$ stands for its euclidean norm. $H^{*}$ is the conjugate transpose of the matrix $H . \mathcal{L}_{2}^{n}$ denotes the space of $\mathbb{R}^{n}$-valued square integrable functions defined on $[0, \infty)$, with norm $\|\cdot\|$, and $\mathcal{L}_{2 e}^{n}$ is the extended $\mathcal{L}_{2}^{n}$ space. Given a bounded system $G$ on $\mathcal{L}_{2}^{n}$, we use $\|G\|$ to denote the induced norm of $G . \mathbf{R H}_{\infty}^{l \times m}$ is used to denote the space of proper rational transfer matrices (of dimension $l \times m$, that will be dropped when there is no ambiguity) with no poles in the closed right half plane. $\widehat{f}(j \omega)$ is the Fourier transform of $f(t)$. The truncation operator $P_{T}$ is defined by $P_{T} f(t)=f(t)$ when $t \leq T$ and $P_{T} f(t)=0$ when $t>T$. If necessary, we use " $\star$ " to denote the transpose of the corresponding symmetric block in a matrix. Let $\mathcal{D}_{h(t)}$ denote the time-delay operator $\mathcal{D}_{h(t)}(v):=v(t-h(t))$, for the case of constant time-delay we will suppress the time dependency on $h(t)$, i.e. $\mathcal{D}_{h}$. Analogously, we define $\mathcal{S}_{h(t)}=\mathcal{D}_{h(t)}-1$. Finally, a minimal state-space realisation of the transfer function $G(s)=C(s I-A)^{-1} B+D$ is denoted by $G \sim\left[\begin{array}{l|l}A & B \\ \hline C & D\end{array}\right]$. 


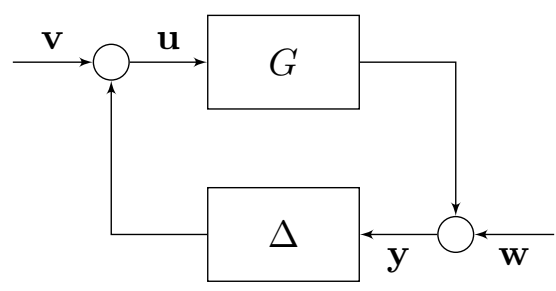

Figure 1. Feedback system $\Sigma(G, \Delta)$.

\section{Preliminaries}

This section serves to introduce some basic notions needed throughout this work. First of all, the IQC framework is briefly presented. The system under consideration is the feedback system $\Sigma(G, \Delta)$ shown in Figure 1, which is given by the following equation

$$
\begin{aligned}
& \mathbf{y}=G \mathbf{u}+\mathbf{w}, \\
& \mathbf{u}=\Delta \mathbf{y}+\mathbf{v},
\end{aligned}
$$

where $\mathbf{w} \in \mathcal{L}_{2 e}^{l}, \mathbf{v} \in \mathcal{L}_{2 e}^{m}$ are exogenous inputs, $G \in \mathbf{R H}_{\infty}^{l \times m}$ and $\Delta$ is a bounded and causal operator. An operator $\Delta$ is causal if $P_{T} \Delta=P_{T} \Delta P_{T}$, and is bounded if its gain $\|\Delta\|$ is finite.

Definition 2.1. The system $\Sigma(G, \Delta)$ is well-posed if the map $(\mathbf{y}, \mathbf{u}) \mapsto(\mathbf{v}, \mathbf{w})$ has a causal inverse on $\mathcal{L}_{2 e}^{l+m}$. That is, for any $\mathbf{w} \in \mathcal{L}_{2 e}^{l}$ and $\mathbf{v} \in \mathcal{L}_{2 e}^{m}$, there exists a solution $\mathbf{y} \in \mathcal{L}_{2 e}^{l}$ and $\mathbf{u} \in \mathcal{L}_{2 e}^{m}$, which depends causally on $\mathbf{v}$ and $\mathbf{w}$.

Definition 2.2. The system $\Sigma(G, \Delta)$ is stable if for any solution, there exists a constant $C>0$ such that

$$
\int_{0}^{T}\left(|\mathbf{y}(t)|^{2}+|\mathbf{u}(t)|^{2}\right) d t \leq C \int_{0}^{T}\left(|\mathbf{v}(t)|^{2}+|\mathbf{w}(t)|^{2}\right) d t
$$

for all $T \geq 0$.

Definition 2.3. Let $\Pi: j \mathbb{R} \mapsto \mathbb{C}^{(l+m) \times(l+m)}$ be a measurable Hermitian valued function. Two signals $\mathbf{y} \in \mathcal{L}_{2}^{l}$ and $\mathbf{u} \in \mathcal{L}_{2}^{m}$ are said to satisfy the IQC defined by $\Pi$ if

$$
\int_{-\infty}^{\infty}\left[\begin{array}{c}
\widehat{\mathbf{y}}(j \omega) \\
\widehat{\mathbf{u}}(j \omega)
\end{array}\right]^{*} \Pi(j \omega)\left[\begin{array}{c}
\widehat{\mathbf{y}}(j \omega) \\
\widehat{\mathbf{u}}(j \omega)
\end{array}\right] d \omega \geq 0
$$

Moreover, a bounded system $\Delta: \mathcal{L}_{2 e}^{l} \mapsto \mathcal{L}_{2 e}^{m}$ is said to satisfy the IQC defined by $\Pi$ if $\mathbf{y}$ and $\Delta \mathbf{y}$ satisfy the IQC defined by $\Pi$ for all $\mathbf{y} \in \mathcal{L}_{2}^{l}$.

The main stability criterion, the so-called IQC Theorem is formulated as follows.

Theorem 2.4 (The IQC Theorem). (Megretski \& Rantzer, 1997) Let $G \in \mathbf{R H}_{\infty}^{l \times m}$ and let $\Delta$ be a bounded causal operator. Suppose

(1) for every $\tau \in[0,1]$, the system $\Sigma(G, \tau \Delta)$ is well-posed;

(2) for every $\tau \in[0,1]$, the IQC defined by $\Pi$ is satisfied by $\tau \Delta$; 
(3) there exists $\epsilon>0$ such that

$$
\left[\begin{array}{c}
G(j \omega) \\
I
\end{array}\right]^{*} \Pi(j \omega)\left[\begin{array}{c}
G(j \omega) \\
I
\end{array}\right] \leq-\epsilon I
$$

for all $\omega \in \mathbb{R}$.

Then, the system $\Sigma(G, \Delta)$ is stable.

The multiplier $\Pi$ is often block decomposed into the form

$$
\Pi=\left[\begin{array}{cc}
\Pi_{11} & \Pi_{12} \\
\star & \Pi_{22}
\end{array}\right],
$$

it is important to note that if $\Pi_{11} \geq 0$ and $\Pi_{22} \leq 0$ and $\Delta$ satisfies the IQC defined by $\Pi$, it implies that $\tau \Delta$ satisfies the same IQC for all $\tau \in[0,1]$.

Assume that $\Delta$ is diagonally structured by $n$ sub-systems $\Delta_{i}, i=1, \ldots, n$; i.e.

$$
\Delta=\operatorname{diag}\left(\Delta_{1}, \ldots, \Delta_{n}\right),
$$

and each $\Delta_{i}$ satisfies the IQC defined by

$$
\Pi_{i}=\left[\begin{array}{cc}
\Pi_{i(11)} & \Pi_{i(12)} \\
\star & \Pi_{i(22)}
\end{array}\right],
$$

where the block structures are consistent with the size of the sub-systems $\Delta_{i}$. Then an IQC for $\Delta$ can be easily defined by composing $\Pi_{i}$ appropriately by

$$
\Pi=\left[\begin{array}{ccc|ccc}
\Pi_{1(11)} & & & \Pi_{1(12)} & & \\
& \ddots & & & \ddots & \\
& & \Pi_{n(11)} & & & \Pi_{n(12)} \\
\hline \star & & & \Pi_{1(22)} & & \\
& \ddots & & & \ddots & \\
& & \star & & & \Pi_{n(22)}
\end{array}\right] .
$$

Note that (4) is a frequency-dependent inequality (FDI) condition over an infinite range of frequencies. An effective method to check such a condition is to translate the FDI condition into an LMI problem via KYP Lemma.

Lemma 2.5 (KYP Lemma). (Rantzer, 1996) Let $\Omega$ be a symmetric matrix. For the system $G(s)=C(s I-A)^{-1} B+D$, where $\operatorname{det}(j \omega I-A) \neq 0, \forall \omega$, the following inequalities are equivalent:

- The following FDI holds:

$$
G(j \omega)^{*} \Omega G(j \omega)<0, \quad \forall \omega \in \mathbb{R} .
$$




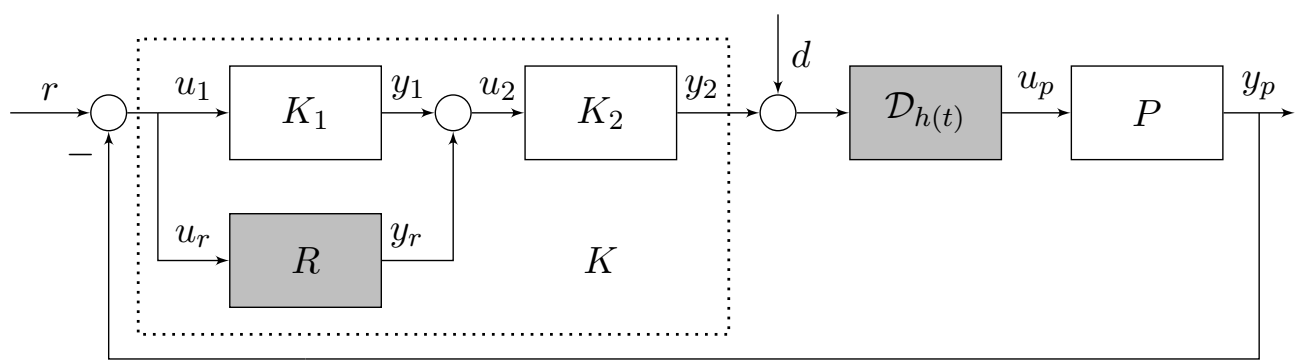

Figure 2. Reset control system setup.

- There exists a matrix $P=P^{\top} \in \mathbb{R}^{n \times n}$, where $n=\operatorname{dim}(A)$, such that

$$
\left[\begin{array}{cc}
A & B \\
I & 0 \\
\hline C & D
\end{array}\right]^{\top}\left[\begin{array}{cc|c}
0 & P & 0 \\
P & 0 & 0 \\
\hline 0 & 0 & \Omega
\end{array}\right]\left[\begin{array}{cc}
A & B \\
I & 0 \\
\hline C & D
\end{array}\right]<0 .
$$

Before closing this section, a definition about passivity is provided, since a passivity property will be used to characterize the input-output behaviour of the reset controller.

Definition 2.6 (van der Schaft (2000)). A system $H: \mathcal{L}_{2 e} \mapsto \mathcal{L}_{2 e}$, with input $u$ and output $y=H u$ is said to be output strictly passive (OSP) if there exist a constant $k_{1}>0$ (excess of output passivity) such that

$$
\int_{0}^{T} u^{\top}(t) y(t) d t \geq k_{1} \int_{0}^{T} y^{\top}(t) y(t) d t
$$

for all functions $u \in \mathcal{L}_{2 e}$ and for all $T \geq 0$.

Note that a system $H$, which is OSP, has a finite gain (Khalil, 2002).

\section{Time-delay reset control systems}

Consider a reset control system as shown in Figure 2, which is formed by a feedback interconnection of a SISO LTI plant $P$, described as

$$
\begin{aligned}
& \dot{\mathbf{x}}_{p}(t)=A_{p} \mathbf{x}_{p}(t)+B_{p} u_{p}(t), \\
& y_{p}(t)=C_{p} \mathbf{x}_{p}(t),
\end{aligned}
$$

and a controller $K$, that is formed by a reset controller $R$ in parallel with a linear controller $K_{1}$, both connected in series with another linear controller $K_{2}$.

Remark 1. This structure of the controller $K$ (see dotted box in Figure 2) encompasses different reset controller previously considered in the literature, see, for example, the proportional integral plus Clegg integrator (PI+CI) (Baños \& Barreiro, 2012) or the approach of using a reset controller in series with an LTI controller (Horowitz \& Rosenbaum, 1975; Zheng, Chait, Hollot, Steinbuch, \& Norg, 2000).

The considered reset controller $R$, consists of an LTI controller together with a reset 
action, it is described by the following impulsive differential equation (IDE)

$$
\begin{array}{ll}
\dot{\delta}(t)=1, \quad \dot{\mathbf{x}}_{r}(t)=A_{r} \mathbf{x}_{r}(t)+B_{r} u_{r}(t), & \left(y_{r}, u_{r}\right) \in S_{F} \text { or } \delta<\delta_{m}, \\
\delta\left(t^{+}\right)=0, \quad \mathbf{x}_{r}\left(t^{+}\right)=0, & \left(y_{r}, u_{r}\right) \in S_{J} \text { and } \delta \geq \delta_{m}, \\
y_{r}(t)=C_{r} \mathbf{x}_{r}(t)+D_{r} u_{r}(t), &
\end{array}
$$

where $S_{F}$ and $S_{J}$ are the flow and jump set, respectively. In addition, zero initial conditions are considered, i.e. $\delta(0)=0$ and $\mathbf{x}_{r}(0)=0$. Time regularisation is necessary to avoid Zeno behaviour (see Nešić, Zaccarian, and Teel (2008) and the references therein), that consists on switching off the reset mechanism for a time interval of length $\delta_{m}>0$ after each reset time.

The two linear controllers $K_{1}$ and $K_{2}$, are given by the following equations

$$
\begin{aligned}
& \dot{\mathbf{x}}_{1}(t)=A_{1} \mathbf{x}_{1}(t)+B_{1} u_{1}(t), \\
& y_{1}(t)=C_{1} \mathbf{x}_{1}(t)+D_{1} u_{1}(t),
\end{aligned}
$$

and

$$
\begin{aligned}
& \dot{\mathbf{x}}_{2}(t)=A_{2} \mathbf{x}_{2}(t)+B_{2} u_{2}(t), \\
& y_{2}(t)=C_{2} \mathbf{x}_{2}(t)+D_{2} u_{2}(t),
\end{aligned}
$$

respectively. As can be seen from Figure 2, the connections between blocks of the reset control system are as follows

$$
\begin{aligned}
& u_{p}(t)=d(t-h(t))+y_{2}(t-h(t)) \\
& u_{2}(t)=y_{r}(t)+y_{1}(t) \\
& u_{1}(t)=u_{r}(t)=e(t)=r(t)-y_{p}(t)
\end{aligned}
$$

Here, it is considered an unknown time-varying delay $h(t)$ satisfying

$$
0 \leq h(t) \leq h_{0}, \quad|\dot{h}(t)| \leq h_{1}, \quad \forall t \geq 0 .
$$

Henceforward, we assume that the reset controller exhibits a certain passivity property, this is stated as follows.

Assumption 1. The reset controller $R$ is output strictly passive (OSP) with an excess of output passivity $k_{1}$.

Remark 2. This assumption is not very restrictive since it is easy to obtain an OSP reset controller. OSP is a key property of reset controllers. The two previous works that study input-output properties of reset systems provide procedures to obtain an OSP reset controller. In Carrasco et al. (2010), it was stated that a linear OSP controller remains OSP when reset actions are applied to it regardless the reset law, whenever the system has a temporal regularisation. Alternatively, it is possible to obtain an OSP reset controller by an adequate reset law and an extra feedforward loop from any given linear controller (Forni et al., 2011). 


\section{Stability analysis via IQC}

The main purpose of this work is to derive input-output stability criteria for time-delay reset control systems, to reach that goal we take advantage of the IQC framework. This section is devoted to the presentation of a loop transformation, classes of IQCs, and stability criteria.

\subsection{Loop transformation}

The IQC framework deals with a wide class of system, that consists of feedback configurations as illustrated in Figure 1, where $G$ is the transfer function of a known LTI system and all uncertainties or nonlinearities affecting the system (the "troublemaking" components, see shadow boxes in Figure 2) are regarded in the operator $\Delta$. In order to apply the IQC Theorem, the reset control system presented in the previous section will be rewritten as a system $\Sigma(G, \Delta)$, according to the setup of Figure 1. A representation in the form $\Sigma(G, \Delta)$ of the reset system presented in Section 3, with a time-varying delay interconnection, is given by

$$
\begin{gathered}
\bar{G}=\left[\begin{array}{cc}
0 & -P \\
K_{2} & -K_{2} K_{1} P
\end{array}\right], \\
\bar{\Delta}=\left[\begin{array}{cc}
R & 0 \\
0 & \mathcal{D}_{h(t)}
\end{array}\right] .
\end{gathered}
$$

An alternative (equivalent) representation in terms of the operator $\mathcal{S}_{h(t)}$ is more convenient to study this system. That is composed by the systems $G$ and $\Delta$ forming a feedback system $\Sigma(G, \Delta)$, where $G$ and $\Delta$ are given by

$$
\begin{gathered}
G=\left[\begin{array}{cc}
-\frac{K_{2} P}{1+K_{2} K_{1} P} & -\frac{P}{1+K_{2} K_{1} P} \\
\frac{K_{2}}{1+K_{2} K_{1} P} & -\frac{K_{2} K_{1} P}{1+K_{2} K_{1} P}
\end{array}\right], \\
\Delta=\left[\begin{array}{cc}
R & 0 \\
0 & \mathcal{S}_{h(t)}
\end{array}\right] .
\end{gathered}
$$

This representation is illustrated in Figure 3.

The well-posedness of reset control systems has been analysed in Baños and Barreiro (2012) and Baños, Mulero, Barreiro, and Davó (2016), and specifically for reset control systems with time-delay in Cánovas, Mulero, and Baños (2016). Since the reset controller $R$, given by (13), contains a time regularisation, then it directly follows that reset instants are well-defined and distinct (that is usually referred to as well-posedness of the reset instants). Thus, since the base system is well-posed, then well-posedness of the reset control system directly follows from the well-posedness of the reset instants. 


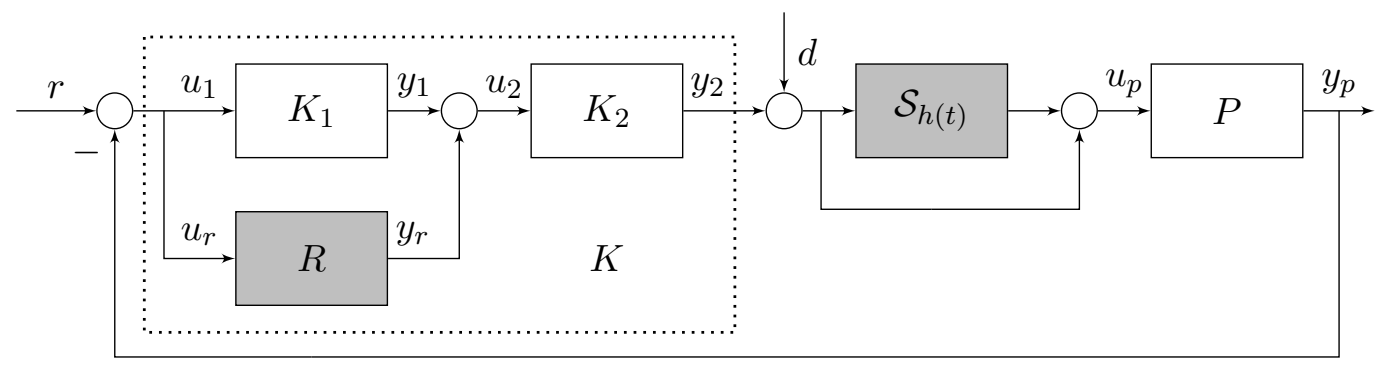

Figure 3. Loop transformation for the considered reset control system.

\subsection{Classes of IQCs}

The IQC Theorem will be applied once the system $\Delta$ is characterized by an appropriate IQC. Different classes of multipliers will be presented ahead.

Definition 4.1. Given a reset controller $R$ satisfying Assumption 1 , $\Pi$ belongs to the class of multipliers $\Pi_{r}$ if there exist $\Upsilon(\omega)=\Upsilon(\omega)^{*}>0$ for all $\omega \in \mathbb{R}$ such that

$$
\Pi=\left[\begin{array}{cc}
0 & 0.5 \Upsilon(\omega) \\
0.5 \Upsilon(\omega) & -k_{1} \Upsilon(\omega)
\end{array}\right]
$$

Proposition 4.2. A reset controller $R$ under Assumption 1 satisfies an IQC defined by $\Pi \in \Pi_{r}$.

Proof. By Assumption 1, the system $R$ is OSP, then there exists $k_{1} \geq 0$ that satisfies

$$
\int_{0}^{T} u_{r}^{\top}(t) y_{r}(t) d t-k_{1} \int_{0}^{T} y_{r}^{\top}(t) y_{r}(t) d t \geq 0
$$

for all functions $u_{r} \in \mathcal{L}_{2 e}$ and all $T \geq 0$. Then, by applying the Parserval's theorem and multiplying by $\Upsilon(\omega)=\Upsilon(\omega)^{*}>0$, it is obtained that $R$ satisfies the IQC defined by $\Pi \in \Pi_{r}$.

Remark 3. One of the main concerns in the study of time-delay reset control systems is the presence of discontinuities in the state (or possibly some states) of the reset controller, and how to deal with them. Here, this issue is somehow circumvented using input-output properties of the reset controllers.

Definition 4.3. Given an operator $\mathcal{S}_{h(t)}$ satisfying conditions (17) with $h_{1}<1, \Pi$ belongs to the class of multipliers $\Pi_{d}$ if there exist $D(\omega)=D^{*}(\omega) \geq 0, \Omega(\omega)=$ $\Omega^{*}(\omega) \leq 0$, and a rational transfer function $W_{v}(j \omega)$ satisfying

$$
\begin{array}{ll}
\left|W_{v}(j \omega)\right|>1+\frac{1}{\sqrt{1-h_{1}}}, & \text { if } h_{0}|\omega|>1+\frac{1}{\sqrt{1-h_{1}}}, \\
\left|W_{v}(j \omega)\right|>h_{0}|\omega|, & \text { if } h_{0}|\omega| \leq 1+\frac{1}{\sqrt{1-h_{1}}},
\end{array}
$$


such that

$$
\Pi=\left[\begin{array}{cc}
W_{v}(j \omega) D(\omega) W_{v}(j \omega)^{*}+\frac{d}{1-d} \Omega(\omega) & \Omega(\omega) \\
\Omega(\omega) & -D(\omega)+\Omega(\omega)
\end{array}\right] .
$$

In Kao and Rantzer (2007), an example of $W_{v}(j \omega)$ that fulfills the latter property is given as

$$
W_{d}(j \omega)=k \frac{h_{0}^{2}(j \omega)^{2}+c h_{0}(j \omega)}{h_{0}^{2}(j \omega)^{2}+a h_{0}(j \omega)+k c},
$$

where $k=1+1 / \sqrt{1-h_{1}}, a=\sqrt{2 k c}$, and $c>0$.

Proposition 4.4 (Kao and Rantzer (2007)). The operator $\mathcal{S}_{h(t)}$ satisfying conditions given in (17) with $h_{1}<1$ satisfies an IQC defined by $\Pi \in \mathbf{\Pi}_{v}$

In the particular case of constant time delay operator, i.e. $h_{1}=0$, less conservative results could be obtained by using the following IQC.

Definition 4.5. Given an operator $\mathcal{S}_{h}$ satisfying conditions (17) with $h_{1}=0, \Pi$ belongs to the class of multipliers $\Pi_{c}$ if there exist $D(\omega)=D^{*}(\omega) \geq 0, \Omega(\omega)=$ $\Omega^{*}(\omega) \leq 0$, and a rational transfer function $W_{c}(j \omega)$ satisfying $\left|W_{c}(j \omega)\right| \geq\left|\mathcal{S}_{h}(j \omega)\right|$, for all $h \leq h_{0}$ and $\omega \in \mathbb{R}$, such that

$$
\Pi=\left[\begin{array}{cc}
W_{c}(j \omega) D(\omega) W_{c}(j \omega)^{*} & \Omega(\omega) \\
\Omega(\omega) & -D(\omega)+\Omega(\omega)
\end{array}\right] .
$$

Proposition 4.6 (Pfifer and Seiler (2015)). The operator $\mathcal{S}_{h}$ satisfying conditions given in (17) with $h_{1}=0$ satisfies an IQC defined by $\Pi \in \boldsymbol{\Pi}_{c}$

An example of $W_{c}(j \omega)$ satisfying properties stated in Definition 4.5 was presented in

$$
W_{c}(j \omega)=2 \frac{(j \omega)^{2} h_{0}^{2}+3.5 j \omega h_{0}+10^{-6}}{(j \omega)^{2} h_{0}^{2}+4.5 j \omega h_{0}+7.1}
$$

The frequency dependent functions that appear in the multipliers defined in the previous section can be parameterised as

$$
\begin{aligned}
& \Upsilon(\omega)=\Lambda_{r}(j \omega)^{*} K_{r} \Lambda_{r}(j \omega), \\
& D(\omega)=\Lambda_{d}(j \omega)^{*} K_{d} \Lambda_{d}(j \omega), \\
& \Omega(\omega)=\Lambda_{o}(j \omega)^{*} K_{o} \Lambda_{o}(j \omega) .
\end{aligned}
$$

where

$$
\Lambda_{\bullet}(j \omega)=\left[\begin{array}{llll}
1 & \frac{1}{j \omega+\alpha_{\bullet}} & \cdots & \frac{1}{\left(j \omega+\alpha_{\bullet}\right)^{n \bullet-1}}
\end{array}\right]^{\top} .
$$

for a given $\alpha_{\bullet}>0$ and $n_{\bullet} \geq 2$. The bullet denotes $r, d$, or $o$. 
After presenting multipliers for the subsystems $R, \mathcal{S}_{h(t)}$, and $\mathcal{S}_{h}$, we will present multipliers for the overall system $\Delta$, that is the system composed by $R$ and $\mathcal{S}_{h(t)}$ (time-varying delay) or $\mathcal{S}_{h}$ (constant delay).

Definition 4.7. Given a particular choice of $\Lambda_{r}, \Lambda_{d}$, and $\Lambda_{o}$, let $\Pi_{1}$ the class of multipliers with structure

$$
\Pi=\Psi_{1}^{*} K_{1} \Psi_{1},
$$

where

$$
K_{1}=\left[\begin{array}{cccc|cccc}
0 & 0 & K_{r} & 0 & 0 & 0 & 0 & 0 \\
0 & K_{d} & 0 & 0 & 0 & 0 & 0 & 0 \\
K_{r} & 0 & -k_{1} K_{r} & 0 & 0 & 0 & 0 & 0 \\
0 & 0 & 0 & -K_{d} & 0 & 0 & 0 & 0 \\
\hline 0 & 0 & 0 & 0 & 0 & 0 & 0 & 0 \\
0 & 0 & 0 & 0 & 0 & \frac{d}{1-d} K_{o} & 0 & K_{o} \\
0 & 0 & 0 & 0 & 0 & 0 & 0 & 0 \\
0 & 0 & 0 & 0 & 0 & K_{o} & 0 & K_{o}
\end{array}\right],
$$

and

$$
\Psi_{1}=\left[\begin{array}{cccc}
\Lambda_{r} & 0 & 0 & 0 \\
0 & \Lambda_{d} W_{v} & 0 & 0 \\
0 & 0 & \Lambda_{r} & 0 \\
0 & 0 & 0 & \Lambda_{d} \\
\hline 0 & 0 & 0 & 0 \\
0 & \Lambda_{o} & 0 & 0 \\
0 & 0 & 0 & 0 \\
0 & 0 & 0 & \Lambda_{o}
\end{array}\right]
$$

where $K_{r}, K_{d}$, and $K_{o}$ are defined by (27). Then, $\Pi \in \Pi_{1}$ if

$$
\begin{aligned}
& {\left[\begin{array}{cc}
A_{r}^{\top} P_{r}+P_{r} A_{r} & P_{r} B_{r} \\
B_{r}^{\top} P_{r} & 0
\end{array}\right]-\left[\begin{array}{ll}
C_{r} & D_{r}
\end{array}\right]^{\top} K_{r}\left[\begin{array}{cc}
C_{r} & D_{r}
\end{array}\right] \leq 0,} \\
& {\left[\begin{array}{cc}
A_{d}^{\top} P_{d}+P_{d} A_{d} & P_{d} B_{d} \\
B_{d}^{\top} P_{d} & 0
\end{array}\right]-\left[\begin{array}{ll}
C_{d} & D_{d}
\end{array}\right]^{\top} K_{d}\left[\begin{array}{ll}
C_{d} & D_{d}
\end{array}\right] \leq 0,} \\
& {\left[\begin{array}{cc}
A_{o}^{\top} P_{o}+P_{o} A_{o} & P_{o} B_{o} \\
B_{o}^{\top} P_{o} & 0
\end{array}\right]+\left[\begin{array}{ll}
C_{o} & D_{o}
\end{array}\right]^{\top} K_{o}\left[\begin{array}{ll}
C_{o} & D_{o}
\end{array}\right] \leq 0,}
\end{aligned}
$$

where $A_{j}, B_{j}, C_{j}, D_{j}$ are state-space matrices of the basis functions $\Lambda_{j}$ for $j=r, d, o$.

Proposition 4.8. Given a system $\Delta$ given by (19) satisfying Assumption 1 and conditions (17) with $h_{0}<1$ and $\Pi \in \Pi_{1}$, then $\Delta$ satisfies the IQC defined by $\Pi$. 
Proof. Using the composition rule (8), we can obtain a class of multipliers $\boldsymbol{\Pi}_{1}$ for the system $\Delta=\operatorname{diag}\left(R, \mathcal{S}_{h(t)}\right)$. This has the following structure

$$
\Pi_{1}=\left[\begin{array}{cccc}
0 & 0 & 0.5 \Upsilon(\omega) & 0 \\
0 & W_{v}(j \omega) D(\omega) W_{v}(j \omega)^{*}+\frac{d}{1-d} \Omega(\omega) & 0 & \Omega(\omega) \\
0.5 \Upsilon(\omega) & 0 & -k_{1} \Upsilon(\omega) & 0 \\
0 & \Omega(\omega) & 0 & -D(\omega)+\Omega(\omega)
\end{array}\right] .
$$

This can be factored using (27) as (29). Conditions (32)-(34), are obtained by KYP Lemma, and ensure that $R$ and $\mathcal{S}_{h(t)}$ satisfy IQCs defined by $\boldsymbol{\Pi}_{r}$ and $\boldsymbol{\Pi}_{v}$, respectively.

Definition 4.9. Given a particular choice of $\Lambda_{r}, \Lambda_{d}$, and $\Lambda_{o}$, let $\Pi_{2}$ the class of multipliers with structure

$$
\Pi=\Psi_{2}^{*} K_{2} \Psi_{2},
$$

where

$$
K_{2}=\left[\begin{array}{cccc|cccc}
0 & 0 & K_{r} & 0 & 0 & 0 & 0 & 0 \\
0 & K_{d} & 0 & 0 & 0 & 0 & 0 & 0 \\
K_{r} & 0 & -k_{1} K_{r} & 0 & 0 & 0 & 0 & 0 \\
0 & 0 & 0 & -K_{d} & 0 & 0 & 0 & 0 \\
\hline 0 & 0 & 0 & 0 & 0 & 0 & 0 & 0 \\
0 & 0 & 0 & 0 & 0 & 0 & 0 & K_{o} \\
0 & 0 & 0 & 0 & 0 & 0 & 0 & 0 \\
0 & 0 & 0 & 0 & 0 & K_{o} & 0 & K_{o}
\end{array}\right]
$$

and

$$
\Psi_{2}=\left[\begin{array}{cccc}
\Lambda_{r} & 0 & 0 & 0 \\
0 & \Lambda_{d} W_{c} & 0 & 0 \\
0 & 0 & \Lambda_{r} & 0 \\
0 & 0 & 0 & \Lambda_{d} \\
\hline 0 & 0 & 0 & 0 \\
0 & \Lambda_{o} & 0 & 0 \\
0 & 0 & 0 & 0 \\
0 & 0 & 0 & \Lambda_{o}
\end{array}\right]
$$

where $K_{r}, K_{d}$, and $K_{o}$ are defined by (27). Then, $\Pi \in \boldsymbol{\Pi}_{\mathbf{2}}$ if LMIs (32)-(34) are satisfied.

Proposition 4.10. Given a system $\Delta$ given by (19) satisfying Assumption 1 and conditions (17) with $h_{0}=0$ and $\Pi \in \Pi_{2}$, then $\Delta$ satisfies the IQC defined by $\Pi$.

Proof. This proof follows analogously to the proof of Proposition 4.8. Hence, it is omitted here. 


\subsection{Stability conditions}

Once the characteristics of $\Delta=\operatorname{diag}\left(R, \mathcal{S}_{h(t)}\right)$ have been described through an IQC, Theorem 2.4 (IQC Theorem) and Lemma 2.5 (KYP Lemma) are applied to the reset control system $\Sigma(G, \Delta)$. The following proposition provides a sufficient condition for the stability of the considered system.

Proposition 4.11 (Stability criterion for time-varying delay case). Assume $\Sigma(G, \tau \Delta)$ is well-posed for every $\tau \in[0,1]$, with $G \in \mathbf{R H}_{\infty}$ and $\Delta$ given by (19) satisfying conditions (17) with $h_{0}<1$. Let us define a minimal state-space representation

$$
\Psi_{1}\left[\begin{array}{c}
G \\
I
\end{array}\right] \sim\left[\begin{array}{c|c}
A_{1} & B_{1} \\
\hline C_{1} & D_{1}
\end{array}\right]
$$

where $\Psi_{1}$ is defined in (31). Then, $\Sigma(G, \Delta)$ is stable, if there exist symmetric matrices $P_{1}$ and $K_{1}$, defined in (30), of adequate dimension such that there exists $\Pi=\Psi_{1}^{*} K_{1} \Psi_{1} \in \Pi_{1}$ and the following LMI holds

$$
\left[\begin{array}{cc}
A_{1}^{\top} P_{1}+P_{1} A_{1} & P_{1} B_{1} \\
B_{1}^{\top} P_{1} & 0
\end{array}\right]+\left[\begin{array}{cc}
C_{1} & D_{1}
\end{array}\right]^{\top} K_{1}\left[\begin{array}{ll}
C_{1} & D_{1}
\end{array}\right] \leq 0 .
$$

Proof. This proof is based on the application of Theorem 2.4. The system was assumed to be well-posed, then the first condition of Theorem 2.4 is satisfied. The second one is satisfied for $\tau=1$ since by assumption $\Pi \in \Pi_{1}$ and for $\tau \in[0,1)$ due to that the lower right corner of $\Pi \in \Pi_{1}$ is negative semi-definite. Finally, Lemma 2.5 states that satisfying LMI (40) is equivalent to the last condition of Theorem 2.4 and can be concluded that the interconnection is stable.

Proposition 4.12 (Stability criterion for constant time-delay case). Assume $\Sigma(G, \tau \Delta)$ is well-posed for every $\tau \in[0,1]$, with $G \in \mathbf{R H}_{\infty}$ and $\Delta$ given by (19) satisfying conditions (17) with $h_{0}=0$. Let us define a minimal state-space representation

$$
\Psi_{2}\left[\begin{array}{c}
G \\
I
\end{array}\right] \sim\left[\begin{array}{c|c}
A_{2} & B_{2} \\
\hline C_{2} & D_{2}
\end{array}\right]
$$

where $\Psi_{2}$ is defined in (38). Then, $\Sigma(G, \Delta)$ is stable, if there exist symmetric matrices $P_{1}$ and $K_{2}$, defined in (37), of adequate dimension such that there exists $\Pi=\Psi_{2}^{*} K_{2} \Psi_{2} \in \Pi_{2}$ and the following LMI holds

$$
\left[\begin{array}{cc}
A_{2}^{\top} P_{2}+P_{2} A_{2} & P_{2} B_{2} \\
B_{2}^{\top} P_{2} & 0
\end{array}\right]+\left[\begin{array}{ll}
C_{2} & D_{2}
\end{array}\right]^{\top} K_{2}\left[\begin{array}{ll}
C_{2} & D_{2}
\end{array}\right] \leq 0 .
$$

Proof. This proof is very similar to the proof of Proposition 4.11. Therefore, it is omitted here. 


\section{Examples}

In this section, the interest of the presented conditions will be evaluated on some numerical examples. For simplicity, the factorization of the multipliers has been done using $\alpha=\alpha_{\bullet}$ and $n=n_{\bullet}$. Recall that the bullet denotes $r, d$, or $o$. The resulting LMIs have been formulated and solved using CVX (Grant \& Boyd, 2015).

Example 5.1. This example analyses the stability of a reset system with uncertain time-varying delay. Let us consider the following plant and linear controllers

$$
P(s)=\frac{1}{s+1}, \quad K_{1}=0.5, \quad K_{2}(s)=\frac{s+0.6}{0.6 s^{2}+1.2 s} .
$$

On the other hand, it is considered the following reset controller $R$

$$
\begin{array}{ll}
\dot{\delta}(t)=1, \quad \dot{x}_{r}(t)=-0.6 x_{r}(t)+e(t), & \left(y_{r}, e\right) \in S_{F} \text { or } \delta<\delta_{m}, \\
\delta\left(t^{+}\right)=0, \quad x_{r}\left(t^{+}\right)=0, & \left(y_{r}, e\right) \in S_{J} \text { and } \delta \geq \delta_{m}, \\
y_{r}(t)=0.6 x_{r}(t) . &
\end{array}
$$

The reset controller $R$ is a first-order reset element (FORE) (whose associated base system has a stable pole), then it is OSP with $k_{1}=1$ since its base is OSP with $k_{1}=1$ Carrasco et al. (2010). This property is hold for any reset law, whenever the system has a temporal regularisation. The considered time-varying delay $h(t)$ satisfies (17) with $h_{0}=0.45$ and $h_{1}=0.45$. Proposition 4.11 guarantees input-output stability for this system using a factorization of the multipliers with $n=3$ and $\alpha=2$. Optimal design of basis functions is still an open question (Veenman, Scherer, \& Köroğlu, 2016). In (Tugal et al., 2016) the authors propose that the selection of the poles can be done by exploring the behaviour of equation (4) for constant multipliers. Then the poles can be included by the range of frequencies where the inequality is not satisfied.

To conclude this example a numerical simulation is performed. For the reset controller $R$, two reset laws are considered:

- Zero-crossing (Z-C). The reset action occurs when the input of the controller is zero, i.e., $e(t)=0$. That is modelled by

$$
\begin{aligned}
& S_{F}=\left\{\left(y_{r}, e\right): e \neq 0\right\}, \\
& S_{J}=\left\{\left(y_{r}, e\right): e=0\right\} .
\end{aligned}
$$

- Variable reset band (VRB). The reset action occurs when $\bar{h} \dot{e}(t)+e(t)=0$, for a given $\bar{h}$. That is defined by

$$
\begin{aligned}
& S_{F}=\left\{\left(y_{r}, e\right): \bar{h} \dot{e}+e \neq 0\right\}, \\
& S_{J}=\left\{\left(y_{r}, e\right): \bar{h} \dot{e}+e=0\right\} .
\end{aligned}
$$

Here, a value of $\bar{h}=0.4$ has been selected, motivated by a time-varying delay $h(t)=0.4+0.1 \sin (t)$ that is used in the simulation.

Time regularisation is considered in both cases. Figure 4 shows the step responses for this control system with reset (Z-C and VRB are considered as reset laws) and without reset action (base). Note how the reset actions improve the performance of this control system, in particular, variable reset band yields the best performance. 


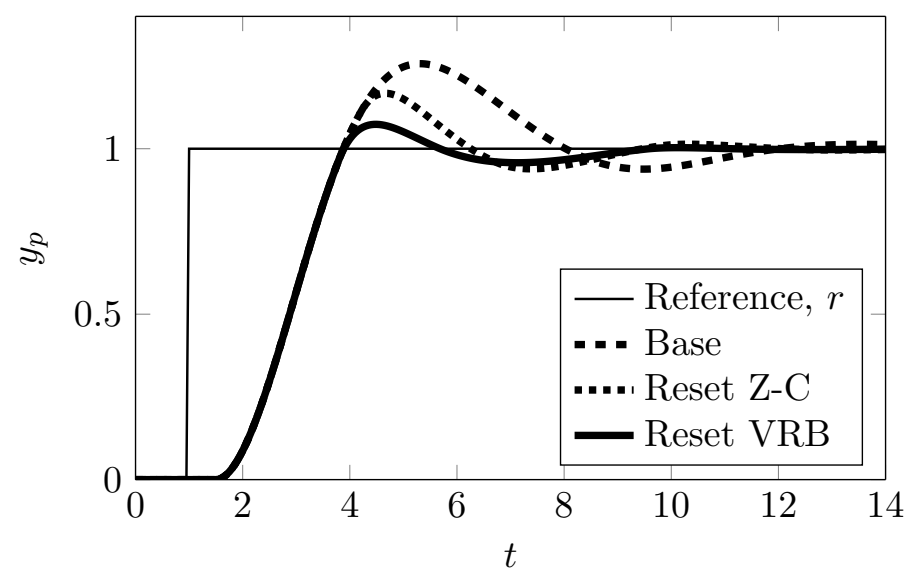

Figure 4. Step reference response in Example 5.1.

Example 5.2. This example consider an unstable base system with constant time delay and a reset strategy that is able to stabilise it. The considered base controller is not OSP, but an adequate reset law and an extra feedforward loop will enforce the OSP property in the reset controller (the procedure presented in Forni et al. (2011) is used here).

We consider a time-delay reset control system according to the setup in Figure 2, with a plant $P$ given by

$$
\begin{aligned}
& \dot{x}_{p}(t)=u_{p}(t), \\
& y_{p}(t)=x_{p}(t),
\end{aligned}
$$

a linear controller $K_{1}$ given by $y_{1}(t)=u_{1}(t)$, a linear controller $K_{2}$ given by $y_{2}(t)=$ $u_{2}(t)$, and a reset controller $R$ described by

$$
\begin{array}{ll}
\dot{\delta}(t)=1, \quad \dot{x}_{r}(t)=x_{r}(t)+e(t), & \left(y_{r}, e\right) \in S_{F} \text { or } \delta<\delta_{m}, \\
\delta\left(t^{+}\right)=0, \quad x_{r}\left(t^{+}\right)=0, & \left(y_{r}, e\right) \in S_{J} \text { and } \delta \geq \delta_{m}, \\
y_{r}(t)=x_{r}(t)+\epsilon_{3} e(t), &
\end{array}
$$

where

$$
\begin{aligned}
& S_{F}=\left\{\left(y_{r}, e\right):\left(e+\epsilon_{1} y_{r}\right)\left(y_{r}-\epsilon_{2} e\right) \geq 0\right\}, \\
& S_{J}=\left\{\left(y_{r}, e\right):\left(e+\epsilon_{1} y_{r}\right)\left(y_{r}-\epsilon_{2} e\right) \leq 0\right\} .
\end{aligned}
$$

This controller has a shortage of input passivity proportional to $\epsilon_{1}$ and $\delta_{m}$ when $\epsilon_{3}=0$ (Forni et al., 2011). In this particular case, taking the values $\epsilon_{1}=0.01, \epsilon_{2}=1.5$ and $\delta_{m}=0.01$, for a value of $\epsilon_{3}=0.031$, it is obtained that it is OSP with $k_{1}=1.37$. Proposition 4.12 guarantees input-output stability for this control system until a value of time delay equal to $0.577,0.579$, and 0.581 , by using $\alpha=2$ and $n=3,4$, and 5 , respectively. Figure 5 shows the step reference response for this control system with reset and without reset action (base), this simulation was carried out for a time-delay $h=0.40$. 


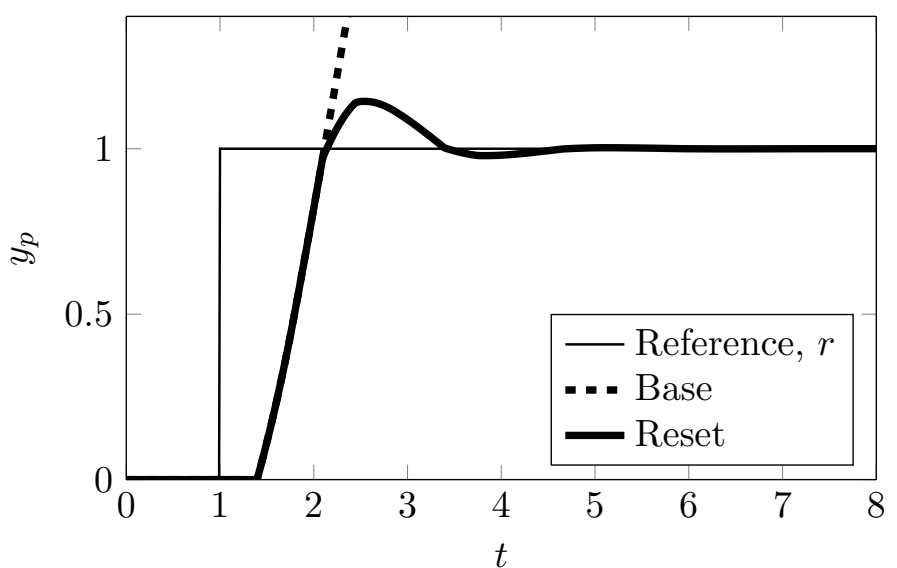

Figure 5. Step reference response in Example 5.2. Note that the unstable response of the base system has been truncated

\section{Conclusion}

This work presents stability criteria for reset control systems in presence of timevarying delay. The obtained criteria are expressed in the form of LMIs. An appealing feature of this approach is that the presence of discontinuities in the state (or possibly some states) of the reset controller is somehow circumvented, by using input-output properties of the reset controllers. In addition, these criteria holds under more general conditions than those imposed by previous works. Various examples illustrate the effectiveness of the approach, in particular, an example shows that reset actions can stabilise an unstable base system. Finally, it should be noted that the approach used in this work may be extended to include uncertain and nonlinear elements.

\section{Acknowledgement}

This work has been supported by Ministerio de Economia e Innovacion of Spain under projects DPI2013-47100-C2-1-P and DPI2016-79278-C2-1-R (including FEDER cofunding). The first author is also supported by an FPU grant (FPU12/01026) from the Ministerio de Educacion, Cultura y Deporte of Spain.

\section{References}

Baños, A., \& Barreiro, A. (2009). Delay-independent stability of reset control systems. IEEE Transactions on Automatic Control, 54, 341-346.

Baños, A., \& Barreiro, A. (2012). Reset control systems. Springer.

Baños, A., Mulero, J. I., Barreiro, A., \& Davó, M. A. (2016). An impulsive dynamical systems framework for reset control systems. International Journal of Control, 89(10), 1985-2007.

Barreiro, A., \& Baños, A. (2010). Delay-dependent stability of reset systems. Automatica, 46, 216-221.

Beker, O., Hollot, C., \& Chait, Y. (2001). Plant with integrator: an example of reset control overcoming limitations of linear feedback. IEEE Transactions on Automatic Control, 46(11), 1797-1799. 
Cánovas, C. D., Mulero, J. I., \& Baños, A. (2016). Well-posedness of reset control systems with input delay. In 20th international conference on system theory, control and computing (pp. 404-409).

Carrasco, J., Baños, A., \& van der Schaft, A. (2010). A passivity-based approach to reset control systems stability. Systems \& Control Letters, 59(1), 18-24.

Clegg, J. C. (1958). A nonlinear integrator for servomechanisms. Transactions of the American Institute of Electrical Engineers, 77, 41-42.

Davó, M. A., \& Baños, A. (2013). Delay-dependent stability of reset control systems with input/output delays. In IEEE 52nd Annual Conference on Decision and Control (pp. 20182023).

Davó, M. A., Gouaisbaut, F., Baños, A., Tarbouriech, S., \& Seuret, A. (2015). Stability of time-delay reset control systems with time-dependent resetting law. IFAC-PapersOnLine, $48(27), 371-376$.

Fetzer, M., \& Scherer, C. W. (2016). A general integral quadratic constraints theorem with applications to a class of sampled-data systems. SIAM Journal on Control and Optimization, $54(3), 1105-1125$.

Forni, F., Nešić, D., \& Zaccarian, L. (2011). Reset passivation of nonlinear controllers via a suitable time-regular reset map. Automatica, 47(9), 2099-2106.

Grant, M., \& Boyd, S. (2015). CVX: Matlab software for disciplined convex programming, version 2.1. http://cvxr.com/cvx.

Horowitz, I. M., \& Rosenbaum, P. (1975). Non-linear design for cost of feedback reduction in systems with large parameter uncertainty. International Journal of Control, 21(6), 9771001.

Kao, C.-Y., \& Rantzer, A. (2007). Stability analysis of systems with uncertain time-varying delays. Automatica, 43(6), $959-970$.

Khalil, H. K. (2002). Nonlinear systems. Prentice Hall.

Krishman, K. R., \& Horowitz, I. M. (1974). Synthesis of a non-linear feedback system with significant plant-ignorance for prescribed system tolerances. International Journal of Control, 19(4), 689-706.

Megretski, A., \& Rantzer, A. (1997). System analysis via integral quadratic constraints. IEEE Transactions on Automatic Control, 42(6), 819-830.

Mercader, P., Carrasco, J., \& Baños, A. (2013). IQC analysis for time-delay reset control systems with first order reset elements. In IEEE 52nd Annual Conference on Decision and Control (p. 2251-2256).

Mercader, P., Davó, M. A., \& Baños, A. (2013). $\mathcal{H}_{\infty} / \mathcal{H}_{2}$ analysis for time-delay reset control systems. In 3rd International Conference on Systems and Control (pp. 518-523).

Nešić, D., Zaccarian, L., \& Teel, A. R. (2008). Stability properties of reset systems. Automatica, $44(8), 2019-2026$.

Pfifer, H., \& Seiler, P. (2015). An overview of integral quadratic constraints for delayed nonlinear and parameter-varying systems. arXiv preprint arXiv:1504.02502.

Rantzer, A. (1996). On the Kalman-Yakubovich-Popov lemma. Systems 85 Control Letters, $28(1), 7-10$.

Tugal, H., Carrasco, J., Falcon, P., \& Barreiro, A. (2016). Stability analysis of bilateral teleoperation with bounded and monotone environments via Zames-Falb multipliers. IEEE Transactions on Control Systems Technology.

van der Schaft, A. (2000). L2-gain and passivity techniques in nonlinear control. Springer London.

Veenman, J., Scherer, C. W., \& Köroğlu, H. (2016). Robust stability and performance analysis based on integral quadratic constraints. European Journal of Control, 31, 1-32.

Zheng, Y., Chait, Y., Hollot, C., Steinbuch, M., \& Norg, M. (2000). Experimental demonstration of reset control design. Control Engineering Practice, 8(2), 113-120. 